\title{
Early Start of Chemotherapy after Resection of Primary Colon Cancer with Synchronous Multiple Liver Metastases: A Case Report
}

\author{
Yoichiro Yoshida Seiichiro Hoshino Hironari Shiwaku \\ Richiko Beppu Shu Tanimura Shinnosuke Tanaka \\ Yuichi Yamashita
}

Department of Gastroenterological Surgery, Fukuoka University

School of Medicine, Fukuoka, Japan

\section{Key Words}

Colorectal cancer · Chemotherapy · Surgery · XELOX

\begin{abstract}
The start of chemotherapy treatment usually requires a delay of about 4 weeks after surgical resection in patients with primary colorectal cancer and synchronous distant metastasis. However, there is no evidence to indicate the required length of this delay interval. In addition, there is a chance that a patient may die because postoperative chemotherapy was not started soon enough and a metastatic tumor was able to develop rapidly. Here, we present a case in which combination chemotherapy with capecitabine and oxaliplatin (XELOX) was started within 1 week after a right hemicolectomy for synchronous multiple liver metastases. To our knowledge, this is the first report of the start of chemotherapy, involving treatments such as folinic acid, fluorouracil, and oxaliplatin (FOLFOX); folinic acid, fluorouracil, and irinotecan (FOLFIRI); and XELOX, within 1 week after a colorectal cancer operation with anastomosis. The findings suggest possible changes in the start time of chemotherapy after surgery in the future.
\end{abstract}

\section{Introduction}

The surgical resection of asymptomatic primary colorectal cancer with unresectable synchronous metastases is controversial. There is no doubt that, among patients with severe intestinal symptoms, resection is mandatory before starting systemic 
chemotherapy [1-3]. Palliative resection of the primary tumor is also reported to improve the efficacy of systemic chemotherapy [4] and prolong the duration of chemotherapy [5]. A recent review article suggested that non-curative resection of asymptomatic colorectal primary tumors may prolong survival in patients with metastatic colorectal cancer [6]. On the other hand, another article concluded that initial chemotherapy should be started, with resection of the primary tumor reserved for the small proportion of patients who develop major complications from the primary tumor, because resection of an asymptomatic primary tumor provides only minimal palliative benefit [7].

The purpose of surgical resection of primary tumors is the prevention of hemorrhage, perforation, and bowel obstruction. In many cases, it is not possible for patients to continue chemotherapy treatments because of complications such as bleeding, perforation, and bowel obstruction after chemotherapy is started without surgical resection of the primary tumor. Thus, it seems necessary to surgically remove the primary tumor in order to continue chemotherapy with few complications. However, surgical resection may delay the start of chemotherapy [8]. Generally, an interval of 4 weeks is considered necessary after an operation before beginning chemotherapy treatments such as folinic acid, fluorouracil, and oxaliplatin (FOLFOX); folinic acid, fluorouracil, and irinotecan (FOLFIRI); and capecitabine and oxaliplatin (XELOX); however, there is no apparent evidence for this delay. A metastatic tumor can enlarge rapidly before the start of chemotherapy and possibly lead to patient death. Because the significance of the postoperative 4-week delay prior to the start of chemotherapy is not clear, we performed an early chemotherapy start in a patient who had undergone a right hemicolectomy for synchronous multiple liver metastases.

\section{Case Report}

A 43-year-old man was referred to our hospital with upper abdominal pain and abdominal fullness. Colonoscopy revealed a nearly obstructing tumor in the ascending colon. Stenosis prevented the endoscope from passing through. The patient needed to be fasted because of the stenosis of the

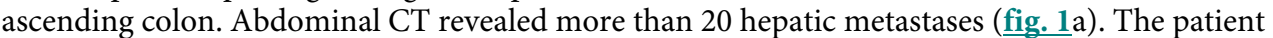
underwent a right hemicolectomy (open surgery) with functional end-to-end anastomosis. The oral side of the tumor was accompanied by significant edema and expansion of the ileum (fig. 2). No drain was used, and the nasogastric tube was removed immediately after surgery. After surgery, the patient was allowed to drink water. On postoperative day 1 , the patient was offered a meal. The postoperative recovery was uneventful. The patient strongly hoped for an early start of chemotherapy on postoperative day 5 . Therefore, we started XELOX therapy on postoperative day 7 [oxaliplatin (130 $\left.\mathrm{mg} / \mathrm{m}^{2}\right)$ on day 1 plus capecitabine $\left(1,000 \mathrm{mg} / \mathrm{m}^{2}\right)$ twice daily on days $1-14$, every 3 weeks]. The patient was subsequently discharged in good condition on postoperative day 13 . XELOX + bevacizumab [BV $(7.5 \mathrm{mg} / \mathrm{kg})$ and oxaliplatin $\left(130 \mathrm{mg} / \mathrm{m}^{2}\right)$ on day 1 plus capecitabine $\left(1,000 \mathrm{mg} / \mathrm{m}^{2}\right)$ twice daily on days $1-14$, every 3 weeks] was administered on postoperative day 28 . After 6 cycles of XELOX + BV, CT revealed a reduction in tumor size (fig. 1b). Laboratory studies revealed a serum carcinoembryonic antigen level of 1,499 ng/ml, which improved to $312 \mathrm{ng} / \mathrm{ml}$ after 6 cycles of chemotherapy. Observed adverse events that had previously appeared included only a peripheral neuropathy in the acute stage (Grade 1) and hand-foot syndrome (Grade 1). Hematological toxicity, surgical site infection, and incisional hernia were not noted.

Over the past 6 months, tumor progression has not occurred; the patient is now undergoing $\mathrm{XELOX}+\mathrm{BV}$ therapy and works daily as a pharmacist. 


\section{Discussion}

The National Comprehensive Cancer Network currently recommends that patients with metastatic colorectal cancer undergo surgical intervention if they have bowel obstruction, an impending obstruction, or metastases that are potentially resectable. Complications from the primary lesion are uncommon in these circumstances, and the removal of the lesion delays initiation of systemic chemotherapy. Resection of colorectal cancer in patients with severe symptoms is mandatory before starting chemotherapy. In the past, some investigators recommended routine resection of the primary tumor in order to prevent the need for urgent surgical procedures because of local complications $[1,2]$. In a study by Ruo et al. [9], 30 (29\%) of the 103 patients who were initially managed without bowel resection required a subsequent operation for palliation of complications. Recently, some authors have suggested elective resection of asymptomatic colorectal cancers in at least a subset of patients with less advanced stage IV disease [3,9]. Other authors have suggested deferring resection of minimally symptomatic colorectal tumors because most of these patients succumb to progressive systemic disease instead of the complications related to the intact primary lesion $[8,10,11]$.

Currently, the precise timing for starting treatment with chemotherapeutic agents prior to and/or after surgery in order to avoid postoperative complications is not clear, but an at least 4 -week interval has been suggested. In most clinical trials, patients who have undergone an operation within 4 weeks of the trial's start are excluded. In Benoist et al.'s study, the mean interval between diagnosis and chemotherapy start was 44 days in the resection group versus 15 days in the chemotherapy group [8]. In this case, although an operation was performed, the interval between diagnosis and chemotherapy start was 17 days. Resection of the primary tumor significantly increases hospital stay and delays the initiation of chemotherapy, although there is no evidence to suggest that this delay is associated with reduced response rates leading to curative resection or reduced survival. However, there is a chance that patients may die if they are not able to start chemotherapy because of the rapid postoperative progression of a metastatic tumor $[12,13]$.

Enhanced recovery after surgery (ERAS) protocols aim at reducing the surgical stress response and optimizing recovery to reduce the length of hospital stay [14]. All elements in ERAS have separately been shown to improve patient outcome. Because ERAS was developed, an early start of chemotherapy after surgery has been enabled. An early start of chemotherapy after surgery may prevent tumor growth.

Resection of colorectal tumors with severe stenosis and bleeding is the first step in treatment in order to prevent the complications related to colorectal tumors. An early start of chemotherapy after surgery may be safe and improve the prognosis of colon cancer patients with synchronous metastases. Prospective studies are needed to confirm the effect and safety of an early start of chemotherapy after surgery. We have already started a new clinical trial to examine patients receiving an early start of chemotherapy. 

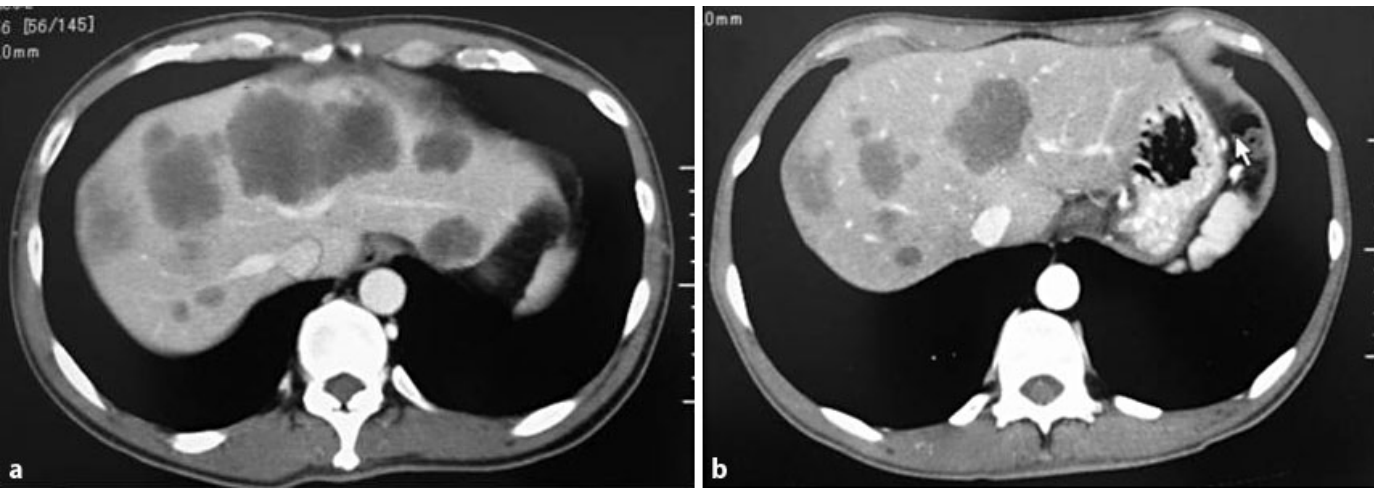

Fig. 1. Abdominal CT. a Imaging before chemotherapy. Multiple liver metastases are visible on the CT scan. b Imaging after 8 cycles of chemotherapy. Partial response can be seen on the CT scan.

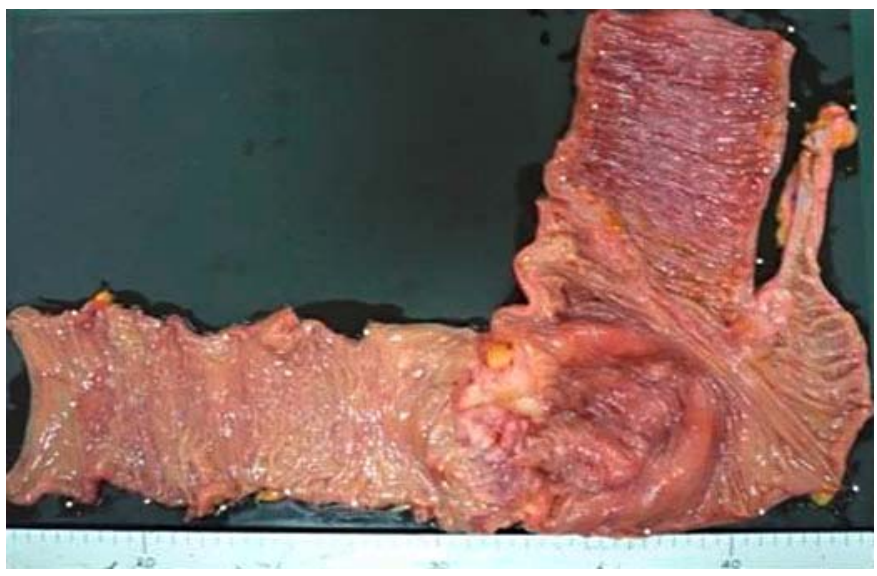

Fig. 2. Resected colon specimen. The resected colon specimen revealed a tumor in the ascending colon. The oral side of the tumor was accompanied by significant edema.

\section{References}

1 Joffe J, Gordon PH: Palliative resection for colorectal carcinoma. Dis Colon Rectum 1981;24:355-360.

2 Longo WE, Ballantyne GH, Bilchik AJ, Modlin IM: Advanced rectal cancer. What is the best palliation? Dis Colon Rectum 1988;31:842-847.

- 3 Rosen SA, Buell JF, Oshida A, Kazsuba S, Hurst R, Michelassi F, Millis JM, Posner MC: Initial presentation with stage IV colorectal cancer: how aggressive should we be? Arch Surg 2000;135:530-534.

4 Temple LK, Hsieh L, Wong WD, Saltz L, Schrag D: Use of surgery among elderly patients with stage IV colorectal cancer. J Clin Oncol 2004;22:3475-3484.

-5 Kaufman MS, Radhakrishnan N, Roy R, Gecelter G, Tsang J, Thomas A, Nissel-Horowitz S, Mehrotra B: Influence of palliative surgical resection on overall survival in patients with advanced colorectal cancer: a retrospective single institutional study. Colorectal Dis 2008;10:498-502.

-6 Eisenberger A, Whelan RL, Neugut AI: Survival and symptomatic benefit from palliative primary tumor resection in patients with metastatic colorectal cancer: a review. Int J Colorectal Dis 2008;23:559-568.

7 Scheer MG, Sloots CE, van der Wilt GJ, Ruers TJ: Management of patients with asymptomatic colorectal cancer and synchronous irresectable metastases. Ann Oncol 2008;19:1829-1835. 
8 Benoist S, Pautrat K, Mitry E, Rouguer P, Penna C, Nordlinger B: Treatment strategy for patients with colorectal cancer and synchronous irresectable liver metastases. Br J Surg 2005;92:1155-1160.

-9 Ruo L, Gougoutas C, Paty PB, Guillem JG, Cohen AM, Wong WD: Elective bowel resection for incurable stage IV colorectal cancer: prognostic variables for asymptomatic patients. J Am Coll Surg 2003;196:722-728.

10 Scoggins CR, Meszoely IM, Blanke CD, Beauchamp RD, Leach SD: Nonoperative management of primary colorectal cancer in patients with stage IV disease. Ann Surg Oncol 1999;6:651-657.

11 Sarela AI, Guthrie JA, Seymourt MT, Ride E, Guillou PJ, O’Riordain S: Non-operative management of the primary tumour in patients with incurable stage IV colorectal cancer. Br J Surg 2001;88:1352-1356.

12 Makino T, Mishima H, Ikenaga M, Tsujinaka T, Takeda M, Mano M: Clinicopathologic features of signet-ring cell carcinoma of the colon and rectum. Jpn J Gastroenterol Surg 2006;39:16-22.

13 Tajima T, Mukai M, Hinoki T, Ootani Y, Sato S, Nakasaki H, Makuuchi H: A case of poorly differentiated carcinoma of the ascending colon with rapid postoperative progression suggesting disseminated carcinomatosis of the bone marrow. Jpn J Gastroenterol Surg 2006;39:265-270.

- 14 Lassen K, Soop M, Nygren J, Cox PB, Hendry PO, Spies C, von Meyenfeldt MF, Fearon KC, Revhaug A, Norderval S, Ljungqvist O, Lobo DN, Dejong CH; Enhanced Recovery After Surgery (ERAS) Group: Consensus review of optimal perioperative care in colorectal surgery: Enhanced Recovery After Surgery (ERAS) Group recommendations. Arch Surg 2009;144:961-969. 